\title{
On the implied market price of risk under the stochastic numéraire *
}

\author{
Nikolai Dokuchaev ${ }^{\dagger}$
}

May 25, 2018

\begin{abstract}
This papers addresses the stock option pricing problem in a continuous time market model where there are two stochastic tradable assets, and one of them is selected as a numéraire. An equivalent martingale measure is not unique for this market, and there are non-replicable claims. Some rational choices of the equivalent martingale measures are suggested and discussed, including implied measures calculated from bond prices constructed as a risk-free investment with deterministic payoff at the terminal time. This leads to possibility to infer a implied market price of risk process from observed historical bond prices.
\end{abstract}

Key words: implied parameters, market price of risk, random numéraire, stochastic bond price, incomplete market

JEL classification: G13

\section{Introduction}

This paper addresses the stock option pricing problem in a continuous time market model. We consider the case where there are two stochastic tradable assets, and one of them is selected as a numéraire. This setting corresponds to a generalized Black-Scholes model where stochastic deviations in the bond prices are allowed. The classical Black and Scholes model includes a bond or money market account with the price $B(t)$ and a single risky asset with the price $S(t)$; the process $B(t)$ is assumed to be non-random or risk-free and is used as a

\footnotetext{
*Annals of Finance. Annals of Finance i Issue 2/2018 DOI: https://doi.org/10.1007/s10436-017-0315-y. Shared link: http://rdcu.be/AedW

${ }^{\dagger}$ Accepted version. Department of Mathematics \& Statistics, Curtin University, GPO Box U1987, Perth, 6845 Western Australia Email: N.Dokuchaev@curtin.edu.au
} 
numéraire, and $S(t)$ is assumed to be a stochastic Itô process with constant volatility. This is a so-called complete market where any claim can be replicated and where there is a unique equivalent martingale (risk-neutral) measure equivalent to the historical measure; the price of a derivative is defined via the expectation of the discounted payoff by this unique equivalent martingale measure.

The pricing of derivatives is usually more difficult for so-called incomplete market models where an equivalent martingale measure is not unique; see, e.g., El Karoui and Quenez (1995).

Some important examples of market incompleteness arise when the money market account is assumed to have a martingale part. Cheng (1991), Kim and Kunitomo (1999), Benninga et al. (2002), and Back (2010), considered an incomplete modification of the Black-Scholes model where $B(t)$ was an Itô process. The cited papers considered martingale pricing method where the option price is calculated as the expectation of the discounted claim under some equivalent risk-neutral measure (martingale measure) such that the discounted stock price $S(t) / B(t)$ is a martingale on a given time interval $[0, T]$ under this measure. Cheng (1991) analyzed only one equivalent martingale measure among all equivalent martingale measures; see Example 1 (iv). Benninga et al. (2002) considered a multi-stock market under requirements that make the choice of an equivalent martingale measure unique in the case of a single stock and stochastic bond; see Example 1(iii). Geman et al. (1995) considered pricing of replicable claims only. Kim and Kunitomo (1999) studied asymptotic properties of this price with respect to a particular equivalent martingale measure. Vecer and $\mathrm{Xu}$ (2004) applied random numéraire to reduce computational dimension for Asian options and general semimartingales. Issaka and SenGupta (2017) applied random numéraire to reduce computational dimension for variance swap options and models based on both Brownian motion and jump processes. In the cited works, the pricing was studied for certain selected measures, and the impact of non-uniqueness of an equivalent martingale measure was not discussed, as well as the presence of non-replicable claims. Some related results and more references can be found in Vecer (2011), Jeanblanc et al (2009), Ch.2, and Schroder (1999).

For portfolio selection problems, related questions arise in the setting with without a random numéraire; see, e.g., Karatzas and Kardaras (2007), Kardaras (2010), Becherer (2010). In addition, a similar setting covers portfolio selection models without riskless assets, including discrete time market models and even single period market models; see, e.g. Won et al (2008).

In this paper, we revisited the pricing problem for options for a market with two tradable stochastic assets. We consider a model with a continuum of different equivalent martingale measures and with claims that cannot be replicated, even when the appreciation rate and 
volatility coefficients are constant (Proposition 2). We investigate the impact of the presence of different equivalent martingale measures and non-replicable claims. Our model is close to the model from Cheng (1991) with a modification that ensures the existence of many equivalent martingale measures. Cheng (1991) studied an impact of the absence of an equivalent martingale measure in the setting with a stochastic bond price $B(t)$ such that $B(T)=1$. In this case, the appreciation rate of the discounted stock price is imploding when terminal time is approached, and the Novikov's condition of existence of an equivalent martingale measure is not satisfied. Our setting removes this feature; we consider a stochastic numéraire without restrictions on the terminal price. This could be close to the model from Cheng (1991) if one considers a stochastic bond with the price $B(t)$ maturing at $T+\varepsilon$, i.e., such that $B(T+\varepsilon)=1$, for an arbitrarily small $\varepsilon>0$. We suggest some criterions of replicability (Theorems 1 and $2)$.

We suggest a parametrisation of the set of equivalent martingale measures that helps to select reasonable equivalent martingale measures, including a measure implied form observed prices of zero coupon bonds (Theorems 7). We discuss some particular choices of these measures, including a measure implied form observed prices of zero coupon bonds and a measure that ensures local risk minimizing hedging strategy (Examples 2-5 and Theorem 8).

Further, we investigate pricing of zero coupon bonds for this market, and derive an equation for the associated risk-free rate or the yield to maturity (Section 5). In this section, we suggest one more approach to selection of equivalent martingale measures. These bonds are auxiliary risk-free assets builded upon one of a risky assets. Using these results, we obtained that, for a market model with stochastic numéraire, it is possible to estimate the market price of risk and therefore the appreciation rate of the stock price process as an implied parameter inferred from a single observed market price of a zero coupon bond, following the classical approach to the so-called implied volatility where the Black-Scholes formula is reversed. This is a novel result, since, as far as we know, this is the first attempt to derive the implied market price of risk based on the bond prices. It appears that estimation of the appreciation rate of stock prices and the market price of risk process from the historical stock prices data is a quite challenging problem that is important for financial applications, especially for optimal portfolio selection. For financial models, estimation of these processes is more difficult than estimation of the volatility since the trend for financial time series is usually relatively small and unstable. Some results and references for the estimation of the appreciation rate and application to portfolio selection can be found in Brennan (1998), Dokuchaev (2005), and Dokuchaev (2002), Ch.9, p.128. Currently, there are few other implied processes considered in the literature, besides the classical implied volatility. Turvey and Komar (2006) consid- 
ered inference of the implied value $a-\theta_{1} \sigma$ from the market option price, in a model that corresponds to a special case of our model with $\widehat{\sigma}=\rho=\widehat{\rho}=0$, presuming that this value is used as the appreciation rate under the pricing measure; for the Black and Scholes model, this value should be the risk-free rate. The implied cumulate risk-free rate was considered in the framework of Black and Scholes model in Dokuchaev (2006) and Hin and Dokuchaev (2014, 2016a,b), as an inferred parameters from stock option prices. Weron (2008) estimated the implied market price of risk for energy prices as the difference between the observable historical Ornstein-Uhlenbeck long term mean and the implied long-term mean infrared from the market options prices. Finally, the implied martingale measure for a bond market was introduced in Bielecki et al. (2009); this construction was based on observation of bond prices for a continuum of maturities. None of these papers considered estimation of the appreciation rate of the stock as an implied parameter inferred from the stock option prices. The present model allows to construct a zero coupon bond as a risk-free investment with deterministic payoff at the terminal time. This leads to the implied market price of risk inferred from the bond prices (equations (30) and (34) below). This gives a possibility to use the observed market bond prices for estimation of the implied market price of risk. This can be a useful addition to the existing methods.

The rest of the paper is organized as follows. In Section 2, we describe the model setting. In Section 3, we discuss replicability of claims and the hedging errors, and we show that the prices can take extreme values for some choices of martingale measures. In Section 4, we discuss possible economically justified choices of the equivalent martingale measures. In Section 5 , we suggest a different choice of the martingale measure implied from the observations of bond prices, and a method of calculation the implied market price of risk for the case of a complete market. In Section 6, parabolic equations are derived for the price, for the hedging strategy, and for the hedging error, in a Markovian setting. The proofs are in the Appendix.

\section{$2 \quad$ Model setting}

It is known that various models financial market lead to different properties with respect to pricing methods, replicability of claims, and arbitrage opportunities. In this section, we describe a market model model consisting of two tradable assets with random continuous in time prices representing a modification of the classical Black-Scholes model where one of the assets is non-random.

We consider a continuous time model of a securities market consisting of two tradable 
assets with the prices $S(t)$ and $B(t), t \geq 0$. The prices evolve as

$$
d S(t)=S(t)(a(t) d t+\sigma(t) d w(t)+\widehat{\sigma}(t) d \widehat{w}(t)), \quad t>0,
$$

and

$$
d B(t)=B(t)(\alpha(t) d t+\rho(t) d w(t)+\widehat{\rho}(t) d \widehat{w}(t)) .
$$

We assume that $W(t)=(w(t), \widehat{w}(t))$ is a standard Wiener process with independent components on a given standard probability space $(\Omega, \mathcal{F}, \mathbf{P})$, where $\Omega$ is a set of elementary events, $\mathbf{P}$ is a probability measure, and $\mathcal{F}$ is a $\mathbf{P}$-complete $\sigma$-algebra of events. The initial prices $S(0)>0$ and $B(0)>0$ are given constants.

We consider this model as an extension of the classical bond and stock market model, where a bond with the price $B(t)$ evolving as

$$
\frac{d B}{d t}(t)=\alpha(t) B(t)
$$

is used as a numéraire. Equation (1) for stochastic numéraire is a generalization of (2); one may say that equation (1) represents a modification of equation for a risk-free asset that takes into account possibility of stochastic disturbances in the return rate. In this setting, $B(t)$ is not exactly a risk-free asset. However, if the processes $\rho(t)$ and $\widehat{\rho}(t)$ are small in some norm, then (1) can be considered as the equation for the money market account with small deviations (see an example in Section 3 below). In particular, the conditions on the coefficients imposed below allow the case where $\rho(t) \equiv \widehat{\rho}(t)=\varepsilon$ for an arbitrarily small $\varepsilon>0$.

If $\operatorname{ess}_{\sup _{t, \omega}}(|\widehat{\sigma}(t, \omega)|+|\widehat{\rho}(t, \omega)|)>0$, we denote by $\left\{\mathcal{F}_{t}\right\}_{t \geq 0}$ the filtration generated by the process $W=(w, \widehat{w})$. If $\operatorname{ess}_{\sup _{t, \omega}}(|\widehat{\sigma}(t, \omega)|+|\widehat{\rho}(t, \omega)|)=0$, we denote by $\left\{\mathcal{F}_{t}\right\}_{t \geq 0}$ the filtration generated by the process $w$ only. In both cases, $\mathcal{F}_{0}$ is trivial, i.e., it is the $\mathbf{P}$-augmentation of the set $\{\emptyset, \Omega\}$.

We assume that the process $\mu(t)=(a(t), \sigma(t), \widehat{\sigma}(t), \alpha(t), \rho(t), \widehat{\rho}(t))$ is $\mathbb{F}$-adapted and bounded.

Let $\widetilde{\sigma} \triangleq \sigma-\rho$ and $\widetilde{\rho} \triangleq \widehat{\sigma}-\widehat{\rho}$. We assume that there exists $c>0$ such that either $|\widetilde{\sigma}(t, \omega)| \geq c$ a.e. or $|\widetilde{\rho}(t, \omega)| \geq c$ a.e..

\section{Discounted stock price and equivalent martingale measures}

Let $\widetilde{S}(t) \triangleq S(t) / B(t)$. By the Itô formula, it follows that this process evolves as

$$
\begin{aligned}
& d \widetilde{S}(t)=\widetilde{S}(t)(\widetilde{a}(t) d t+\widetilde{\sigma}(t) d w(t)+\widetilde{\rho}(t) d \widehat{w}(t)), \\
& \widetilde{S}(0)=S(0) / B(0),
\end{aligned}
$$


where

$$
\widetilde{a} \triangleq a-\alpha+\rho^{2}+\widehat{\rho}^{2}-\sigma \rho-\widehat{\sigma} \widehat{\rho} .
$$

Let $V(t)=\left(V_{1}(t), V_{2}(t)\right)^{\top}=(\widetilde{\sigma}(t), \widetilde{\rho}(t))^{\top}$ and $\widehat{V}(t)=\left(\widehat{V}_{1}(t), \widehat{V}_{2}(t)\right)^{\top}=(\rho(t), \widehat{\rho}(t))^{\top}$. These processes take values in $\mathbf{R}^{2}$. By the assumptions, $\operatorname{essinf}_{t, \omega}|V(t, \omega)|>0$.

Definition 1 Let $\mathcal{T}$ be the set of $\mathcal{F}_{t}$-adapted processes $\theta(t)=\left(\theta_{1}(t), \theta_{2}(t)\right)^{\top}$ with values in $\mathbf{R}^{2}$ such that $\theta_{1}(t) \widetilde{\sigma}(t)+\theta_{2}(t) \widetilde{\rho}(t)=\widetilde{a}(t)$, i.e., $V(t)^{\top} \theta(t)=\widetilde{a}(t)$, and such that

$$
\underset{\omega}{\operatorname{ess} \sup } \int_{0}^{T}|\theta(t)|^{2} d t<+\infty
$$

Here and below $|\cdot|$ is the Euclidean norm of vectors.

Up to the end of this paper, we use notation $\theta$ for elements of the set $\mathcal{T}$ only.

For $\theta \in \mathcal{T}$, set

$$
\mathcal{Z}_{\theta}=\exp \left(-\int_{0}^{T} \theta(s)^{\top} d W(s)-\frac{1}{2} \int_{0}^{T}|\theta(s)|^{2} d s\right)
$$

Our standing assumptions imply that $\mathbf{E} \mathcal{Z}_{\theta}=1$. Define the probability measure $\mathbf{P}_{\theta}$ by $d \mathbf{P}_{\theta} / d \mathbf{P}=\mathcal{Z}_{\theta}$; this measure is equivalent to the measure $\mathbf{P}$. Let $\mathbf{E}_{\theta}$ be the corresponding expectation.

Let

$$
W_{\theta}(t)=\left(\begin{array}{l}
W_{\theta 1}(t) \\
W_{\theta 2}(t)
\end{array}\right)=\int_{0}^{t} \theta(s) d s+W(t) .
$$

By Girsanov's Theorem, $W_{\theta}$ is a standard Wiener process in $\mathbf{R}^{2}$ under $\mathbf{P}_{\theta}$.

For $\theta \in \mathcal{T}$, equation (4) can be rewritten as

$$
d \widetilde{S}(t)=\widetilde{S}(t) V(t)^{\top} d W_{\theta}(t)
$$

Remark 1 Clearly, the set $\mathcal{T}$ has more than one element; it is a linear manifold. Therefore, the selection of the process $\theta(t)$ and the measure $\mathbf{P}_{\theta}$, is not unique.

Example 1 (i) If $\widetilde{\rho} \equiv 0$, then the process $\theta_{1}(t)$ is uniquely defined as $\theta_{1}(t)=\widetilde{\sigma}(t)^{-1} \widetilde{a}(t)$ and is called the marked price of risk process. If, in addition, the process $\widetilde{\sigma}(t)$ is nonrandom, then the process $\widetilde{S}(t)$ has the same distribution under $\mathbf{P}_{\theta}$ for all $\theta \in \mathcal{T}$.

(ii) If $\widetilde{\sigma} \equiv 0$, then the process $\theta_{2}(t)$ is uniquely defined as $\theta_{2}(t)=\widetilde{\rho}(t)^{-1} \widetilde{a}(t)$. 
(iii) Benninga et al. (2002) considered a multi-stock market with special requirements for the equivalent martingale measure. For our special case of a single stock and a stochastic numéraire, these requirements leads to a unique equivalent martingale measure such that the process $\left(\widetilde{S}(t), \exp \left(\int_{0}^{t} k(s) d s\right) B(t)^{-1}\right)$ is a martingale, for a given process $k(t) \geq 0$.

(iv) Let $z(t)=\int_{0}^{t}|V(s)|^{-1} V(s)^{\top} d W(s)$; by Lévy's characterization theorem, it is an onedimensional Wiener process. Let $q(t)=|V(s)|^{-1} \widetilde{a}(t)$. By the assumptions, it is a bounded process. Let $\widehat{z}(t)=\int_{0}^{t} q(s) d s+z(t)$. We have that $V(t)^{\top} d W(t)=|V(t)| d z(t)$ and

$$
d \widetilde{S}(t)=\widetilde{S}(t)(\widetilde{a}(t) d t+|V(t)| d z(t))=\widetilde{S}(t)|V(t)| d \widehat{z}(t) .
$$

By Girsanov's Theorem, there is an equivalent martingale measure $\widehat{\mathbf{P}}$ such that $\widehat{z}(t)$ is a Wiener process under $\widehat{\mathbf{P}}$; in this case, $\widetilde{S}(t)$ is a martingale under $\widehat{\mathbf{P}}$. This martingale measure was studied in Cheng (1991).

Let $\mathcal{Y}_{\theta}$ be the set of all $\mathcal{F}_{t}$-adapted measurable processes with values in $\mathbf{R}^{2}$ that are square integrable on $[0, T] \times \Omega$ with respect to $\ell_{1} \times \mathbf{P}_{\theta}$, where $\ell_{1}$ is the Lebesgue measure.

Let $\mathcal{H}_{\theta}$ be the Hilbert space formed as the completion of the set of $\mathcal{F}_{t}$-adapted measurable processes $y(t)$ such that $\|y\|_{\mathcal{H}_{\theta}}=\left(\mathbf{E}_{\theta} \int_{0}^{T}|\widetilde{S}(t) y(t)|^{2} d t\right)^{1 / 2}<+\infty$.

\section{Wealth and discounted wealth}

Let $X(0)>0$ be the initial wealth at time $t=0$ and let $X(t)$ be the wealth at time $t>0$.

We assume that the wealth $X(t)$ at time $t \geq 0$ is

$$
X(t)=\beta(t) B(t)+\gamma(t) S(t) .
$$

Here $\beta(t)$ is the quantity of the numéraire portfolio, $\gamma(t)$ is the quantity of the stock portfolio, $t \geq 0$. The pair $(\beta(\cdot), \gamma(\cdot))$ describes the state of the securities portfolio at time $t$. Each of these pairs is called a strategy.

Definition 2 Let $\theta \in \mathcal{T}$. A pair $(\beta(\cdot), \gamma(\cdot))$ is said to be an admissible strategy under $\mathbf{P}_{\theta}$ if the processes $\beta(t)$ and $\gamma(t)$ are progressively measurable with respect to the filtration $\left\{\mathcal{F}_{t}\right\}_{t \geq 0}$ and such that

$$
\mathbf{E}_{\theta} \int_{0}^{T} \widetilde{S}(t)^{2} \gamma(t)^{2} d t<+\infty
$$


Definition 3 Let $\theta \in \mathcal{T}$ be given. A pair $(\beta(\cdot), \gamma(\cdot))$ that is an admissible strategy under $\mathbf{P}_{\theta}$ is said to be a self-financing strategy, if there exists a sequence of Markov times $\left\{T_{k}\right\}_{k=1}^{\infty}$ with respect to $\left\{\mathcal{F}_{t}\right\}_{t \geq 0}$ such that $0 \leq T_{k} \leq T_{k+1} \leq T$ for all $k, T_{k} \rightarrow T$ as $k \rightarrow+\infty$ a.s. and that the following holds:

(i) For $k=1,2, .$. ,

$$
\mathbf{E}_{\theta} \int_{0}^{T_{k}}\left(\beta(t)^{2} B(t)^{2}+S(t)^{2} \gamma(t)^{2}\right) d t<+\infty
$$

(ii) The corresponding wealth $X(t)=\gamma(t) S(t)+\beta(t) B(t)$ is such that

$$
d X(t)=\gamma(t) d S(t)+\beta(t) d B(t) .
$$

It be noted that condition (i) in Definition 3 ensures that the stochastic differentials in condition (ii) here are well defined.

The process $\widetilde{X}(t) \triangleq X(t) / B(t)$ is said to be the discounted wealth.

The following lemma is known; see, e.g., Geman et al. (1995), Jamshidian (2008), Bielecki et al. (2009).

Lemma 1 If a strategy $(\beta(t), \gamma(t))$ is self-financing and admissible under $\mathbf{P}_{\theta}$ for some $\theta \in \mathcal{T}$, then, for the corresponding discounted wealth,

$$
d \widetilde{X}(t)=\gamma(t) d \widetilde{S}(t)
$$

Remark 2 Since we assume that the coefficients for the equations for $S(t)$ and $B(t)$ are bounded, it follows from Lemma 1 that if (8) holds for some $\theta$ then $\mathbf{E}_{\theta} \widetilde{X}(T)^{2}<+\infty$ for this $\theta$.

Lemma 2 For every $\theta \in \mathcal{T}$, the processes $\widetilde{X}(t)$ and $\widetilde{S}(t)$ are martingales under $\mathbf{P}_{\theta}$ with respect to $\left\{\mathcal{F}_{t}\right\}_{t \geq 0} t$, i.e., $\mathbf{E}_{\theta}\left\{\widetilde{S}(T) \mid \mathcal{F}_{t}\right\}=\widetilde{S}(t)$ and $\mathbf{E}_{\theta}\left\{\widetilde{X}(T) \mid \mathcal{F}_{t}\right\}=\widetilde{X}(t)$.

Remark 3 Consider an European option with the payoff $B(T) \xi$, where $\xi$ is an $\mathcal{F}_{T}$-measurable random variable. For any $\theta \in \mathcal{T}$ such that $\mathbf{E}_{\theta} \xi^{2}<+\infty$, the option price $\mathbf{E}_{\theta} \xi$ is an arbitragefree price.

\section{Replication of claims: strategies and hedging errors}

In financial mathematics, the most common approach to option pricing is representing the prices as the minimal initial wealth that can be raised, via self-financing strategies, into the 
wealth allowing to fulfil option obligations. This reduces the pricing problem to analysis of replicability of random contingent claims. This section describes certain features of the claim replication for the model described above.

For $\theta \in \mathcal{T}$, let $\mathcal{X}_{\theta}$ be the subspace of $L_{2}\left(\Omega, \mathcal{F}_{T}, \mathbf{P}_{\theta}\right)$ consisting of all $\zeta \in L_{2}\left(\Omega, \mathcal{F}_{T}, \mathbf{P}_{\theta}\right)$ such that there exists an admissible self-financing strategy $(\beta(\cdot), \gamma(\cdot))$ under $\mathbf{P}_{\theta}$ and the corresponding wealth process $X(t)$ such that $X(0)=0$ and $X(T)=B(T) \zeta$.

Let

$$
\mathcal{X}_{\theta}^{\perp} \triangleq\left\{\eta \in L_{2}\left(\Omega, \mathcal{F}_{T}, \mathbf{P}_{\theta}\right): \mathbf{E}_{\theta} \eta=0, \mathbf{E}_{\theta}[\zeta \eta]=0 \text { for all } \zeta \in \mathcal{X}_{\theta}\right\}
$$

Let $\xi \in L_{2}\left(\Omega, \mathcal{F}_{T}, \mathbf{P}_{\theta}\right)$. By the Martingale Representation Theorem, we have that, for some uniquely defined $U_{\theta} \in \mathcal{Y}_{\theta}$ and $c_{\theta} \in \mathbf{R}$,

$$
\xi=c_{\theta}+\int_{0}^{T} U_{\theta}(t)^{\top} d W_{\theta}(t) .
$$

In addition, it follows from the properties of closed subspaces in Hilbert spaces that $\xi$ can be represented via Föllmer-Schweizer decomposition

$$
\xi=c_{\theta}+I_{\theta}+R_{\theta}
$$

Here $c_{\theta}=\mathbf{E}_{\theta} \xi, R_{\theta} \in \mathcal{X}_{\theta}^{\perp}$, and

$$
I_{\theta}=\int_{0}^{T} \gamma_{\theta}(t) d \widetilde{S}(t) \in \mathcal{X}_{\theta}
$$

for some $\gamma_{\theta} \in \mathcal{H}_{\theta}$, i.e., it is the terminal discounted wealth $\widetilde{X}(T)$ for some admissible selffinancing strategy $\left(\beta_{\theta}(\cdot), \gamma_{\theta}(\cdot)\right)$ under $\mathbf{P}_{\theta}$ and for the initial wealth $X(0)=0$. Therefore, a contingent claim $B(T) \xi$ can be decomposed as $B(T)\left(\widetilde{\xi}_{\theta}+R_{\theta}\right)$, where $B(T) \widetilde{\xi}_{\theta}$ is a replicable part such that $\widetilde{\xi}_{\theta}=c_{\theta}+\int_{0}^{T} \gamma_{\theta}(t) d \widetilde{S}(t)$.

We regard the process $\gamma_{\theta}(t)$ here as the hedging strategy, we regard $B(T) R_{\theta}$ as the hedging error, and we regard $R_{\theta}$ as the discounted hedging error. We could regard the value $c_{\theta}=\mathbf{E}_{\theta} \xi$ as a price of an option with the payoff $B(T) \xi$ given that either this price does not depend on the choice of $\theta$ or it is calculated under some reasonable choice of $\theta$; some choices of $\theta$ are discussed in Example 1 and in Section 4 below.

Let us express $\gamma_{\theta}$ via $U_{\theta}$.

Proposition 1 Let $\xi \in L_{2}\left(\Omega, \mathcal{F}_{T}, \mathbf{P}_{\theta}\right)$, and let $U_{\theta}$ be defined by (10). Let

$$
\nu_{\theta}(t)=\frac{U_{\theta}(t)^{\top} V(t)}{|V(t)|^{2}}, \quad \eta_{\theta}(t)=U_{\theta}(t)-\nu_{\theta}(t) V(t) .
$$


Then (11) holds with

$$
I_{\theta}=\int_{0}^{T} \nu_{\theta}(t) V(t)^{\top} d W_{\theta}(t), \quad R_{\theta}=\int_{0}^{T} \eta_{\theta}(t)^{\top} d W_{\theta}(t)
$$

In addition,

$$
\left|U_{\theta}(t)\right|^{2} \equiv\left|\nu_{\theta}(t) V(t)\right|^{2}+\left|\eta_{\theta}(t)\right|^{2}, \quad \eta_{\theta}(t)^{\top} V(t) \equiv 0, \quad \mathbf{E}_{\theta} I_{\theta} R_{\theta}=0,
$$

and (12) holds with

$$
\gamma_{\theta}(t)=\nu_{\theta}(t) \widetilde{S}(t)^{-1}
$$

Proof. It suffices to observe that $\eta_{\theta} \in \mathcal{Y}_{\theta}$, and that $\nu_{\theta} V$ is the projection of $U_{\theta}$ on $V$. In particular, it follows that $R_{\theta} \in \mathcal{X}_{\theta}^{\perp}$. The uniqueness follows from the properties of orthogonal subspaces of a Hilbert space.

\section{Some cases of non-replicability and replicability}

The following statement follows from the non-uniqueness of the equivalent martingale measures and The 2nd Fundamental Theorem of Asset Pricing.

Proposition 2 Assume that $\widetilde{\rho}(\cdot) \neq 0$, i.e., it is not an identically zero process. Then the set $\mathcal{X}_{\theta}^{\perp}$ contains non-zero elements, i.e., $\sup _{\eta \in \mathcal{X}_{\theta}^{\perp}} \mathbf{E}_{\theta}|\eta|>0$.

By this proposition, the discounted hedging error $R_{\theta}$ is non-zero in the general case. In other word, a contingent claim of a general type is not replicable. For the sake of completeness, we will give in the Appendix the proof adjusted to our model.

Let us describe some cases of replicability.

Let $\left\{\mathcal{F}_{t}^{w}\right\}_{t \geq 0}$ be the filtration generated by the process $w(t)$, and let $\left\{\mathcal{F}_{t}^{\widetilde{S}}\right\}_{t \geq 0}$ be the filtration generated by the process $\widetilde{S}(t)$.

Theorem 1 Assume that the processes $\widetilde{\sigma}(t)$ and $\widetilde{\rho}(t)$ are non-random. Then the claims $B(T) \xi$ are replicable for $\xi \in L_{2}\left(\Omega, \mathcal{F}_{T}^{\widetilde{S}}, \mathbf{P}_{\theta}\right)$ for any $\theta \in \mathcal{T}$. More precisely, there exists an $\mathcal{F}_{t}$-adapted process $\gamma(t)$ such that $\mathbf{E}_{\theta} \int_{0}^{T} \gamma(t)^{2} \widetilde{S}(t)^{2} d t<+\infty$ and $\xi=\mathbf{E}_{\theta} \xi+\int_{0}^{T} \gamma(t) d \widetilde{S}(t)$.

\section{The case of complete market}

Note that the market described in Theorem 1 is incomplete since there are claims that cannot be replicated. The following theorem describes an important special case when the market is complete. 
Theorem 2 Assume that the processes $\widetilde{a}(t)$ and $\widetilde{\sigma}(t)$ are adapted to the filtration $\left\{\mathcal{F}_{t}^{w}\right\}_{t \geq 0}$ generated by the process $w(t)$, and that $\widetilde{\rho}(t) \equiv 0$, i.e., it is an identically zero process. Then $\theta_{1}(t)=\widetilde{a}(t) \widetilde{\sigma}(t)^{-1}$ for any $\theta \in \mathcal{T}$, and the claims $B(T) \xi$ are replicable for $\xi \in L_{2}\left(\Omega, \mathcal{F}_{T}^{w}, \mathbf{P}_{\theta}\right)$.

Corollary 1 Assume that either conditions of Theorem 1 or conditions of Theorem 2 hold. Then the choice of the hedging (replicating) strategy $\gamma$ is unique, i.e., it is the same for all $\theta \in \mathcal{T}$ such that $\mathbf{E}_{\theta} \xi^{2}<+\infty$; the expectation $\mathbf{E}_{\theta} \xi$ is also the same for all these $\theta$.

\section{On relativity of the price and the hedging error}

The following theorems demonstrate that this price $c_{\theta}=\mathbf{E}_{\theta} \xi$ can be selected quite arbitrarily even for the case of an arbitrarily small stochastic deviations in (1), i.e., for arbitrarily small processes $\rho(t)$ and $\widehat{\rho}(t)$. For instance, we can select $\rho(t) \equiv \widehat{\rho}(t)=\varepsilon$ for an arbitrarily small $\varepsilon>0$. This means that the presence of small deviations in (1) changes dramatically the properties of the market model.

We denote $x^{+}=\max (0, x)$ for $x \in \mathbf{R}$.

Theorem 3 Assume that

$$
\underset{t, \omega}{\operatorname{essinf}}|\widetilde{\sigma}(t, \omega) \widehat{\rho}(t, \omega)-\rho(t, \omega) \widetilde{\rho}(t, \omega)|>0
$$

Let $\kappa \in(0,+\infty)$ be given, and let $\xi=B(T)^{-1}(\kappa-S(T))^{+}$. Then the following holds.

(i) For any $\varepsilon>0$, there exists $\theta \in \mathcal{T}$ such that $c_{\theta}=\mathbf{E}_{\theta} \xi \in[0, \varepsilon]$.

(ii) For any $M>0$, there exists $\theta \in \mathcal{T}$ such that $c_{\theta}=\mathbf{E}_{\theta} \xi \geq M$.

Theorem 4 Assume that (16) holds. Let $\kappa \in(0,+\infty)$ be given, and let $\xi=B(T)^{-1}(S(T)-$ $\kappa)^{+}$. Then the following holds.

(i) For any $\varepsilon>0$, there exists $\theta \in \mathcal{T}$ such that $c_{\theta}=\mathbf{E}_{\theta} \xi \in[0, \varepsilon]$.

(ii) For any $\varepsilon>0$, there exists $\theta \in \mathcal{T}$ such that $c_{\theta}=\mathbf{E}_{\theta} \xi \in[S(0)-\varepsilon, S(0)]$.

Consider a strategy that replicates the claim $B(T)\left(c_{\theta}+I_{\theta}\right)$, where $c_{\theta} \in \mathbf{R}$ and $I_{\theta} \in \mathcal{X}_{\theta}$ are such that (11) holds with the discounted hedging error $R_{\theta} \in \mathcal{X}_{\theta}^{\perp}$.

The following theorems show that, for any given $\theta$, the value of the second moment of $R_{\theta}$ is varying widely if it is calculated with respect to other equivalent martingale measures and can take extreme values. 
Theorem 5 Let $\xi$ be a random claim such that (11) holds for some $\theta \in \mathcal{T}, c_{\theta} \in \mathbf{R}, I_{\theta} \in \mathcal{X}_{\theta}$, and $R_{\theta} \in \mathcal{X}_{\theta}^{\perp}$ such that $\mathbf{E}_{\theta} R_{\theta}^{2}>0$. Assume that (10) holds for $U_{\theta} \in \mathcal{Y}_{\theta}$ such that

$$
\underset{\omega}{\operatorname{ess} \sup } \int_{0}^{T}\left|U_{\theta}(t, \omega)\right|^{2} d t<+\infty, \quad \underset{\omega}{\operatorname{essinf}} \int_{0}^{T}\left|\eta_{\theta}(t, \omega)\right| d t>0,
$$

where $\eta_{\theta}$ is defined by (13). Then, for any $M>0$, there exists $\vartheta \in \mathcal{T}$ such that $\mathbf{E}_{\vartheta} R_{\theta}^{2} \geq M$.

Theorem 6 Let $\xi$ be a random claim such that (11) holds for some $\theta \in \mathcal{T}, c_{\theta} \in \mathbf{R}, I_{\theta} \in \mathcal{X}_{\theta}$, and $R_{\theta} \in \mathcal{X}_{\theta}^{\perp}$ such that $\mathbf{E}_{\theta} R_{\theta}^{2}>0$. Assume that (10) holds for $U_{\theta} \in \mathcal{Y}_{\theta}$ such that

$$
\underset{t, \omega}{\operatorname{ess} \sup }\left|U_{\theta}(t, \omega)\right|<+\infty, \quad \underset{t, \omega}{\operatorname{essinf}}\left|\eta_{\theta}(t, \omega)\right|>0,
$$

where $\eta_{\theta}$ is defined by (13). Then, for any $\varepsilon>0$, there exists $\vartheta \in \mathcal{T}$ such that $\mathbf{E}_{\vartheta} R_{\theta}^{2} \leq \varepsilon$.

It can be noted that it is not uncommon to observe pricing abnormalities in meaningful market models; see e.g. Ruf (2013) and the references therein.

To overcome relativity of pricing outlined in Theorems 3-5, we need to investigate reasonable choices for the martingale measures.

\section{On selection of $\theta$ and the equivalent martingale measure}

We have found that, for any $\theta \in \mathcal{T}$, the discounted prices process $\widetilde{S}$ is a martingale under the measure $\mathbf{P}_{\theta}$. Therefore, in a general case, there are many equivalent martingale measures. A question arises which particular $\theta$ should be used for pricing of options. In the literature, there are many methods developed for this problem, mainly for the incomplete market models with random volatility and appreciation rate.

One may look for "optimal" $\theta$ and $c_{\theta}$ in the spirit of mean-variance pricing, such that $\mathbf{E} R_{\theta}^{2}$ is minimal; see, e.g., Schweizer (2001). A generalization of this approach leads to minimization of $\mathbf{E}\left|R_{\theta}\right|^{q}$ for $q \geq 1$. An alternative approach is to define the price as $\sup _{\theta \in \mathcal{T}_{0}} c_{\theta}$ for some reasonably selected set $\mathcal{T}_{0} \subset \mathcal{T}$. In particular, this pricing rule leads to a corrected volatility smile in the case of an incomplete market with random volatility (Dokuchaev (2011)).

The following theorem provides an unifying approach for selection of $\theta$. In particular, this approach allows to include models listed in Example 1.

Theorem 7 Let $\theta=\left(\theta_{1}, \theta_{2}\right)^{\top} \in \mathcal{T}$ be given, and let $\varrho(t)=\widehat{V}(t)^{\top} \theta(t)$, i.e.,

$$
\begin{aligned}
& \widetilde{\sigma} \theta_{1}+\widetilde{\rho} \theta_{2}=\widetilde{a}, \\
& \rho \theta_{1}+\widehat{\rho} \theta_{2}=\varrho .
\end{aligned}
$$


Then

$$
\begin{aligned}
& d S(t)=S(t)\left([a(t)-\widetilde{a}(t)-\varrho(t)] d t+\sigma(t) d W_{1 \theta}(t)+\widehat{\sigma}(t) d W_{2 \theta}(t)\right), \\
& \left.d B(t)=B(t)\left([\alpha(t)-\varrho(t)] d t+\rho(t) d W_{1 \theta}(t)\right)+\widehat{\rho}(t) d W_{2 \theta}(t)\right) .
\end{aligned}
$$

Theorem 7 gives a parametrization of the set $\mathcal{T}$ via $\varrho$.

Examples 2-5 below demonstrate how this parametrization helps to identify some reasonable choices of $\theta$. For these examples, we assume that

$$
\underset{t, \omega}{\operatorname{essinf}}|\widetilde{\sigma}(t, \omega) \widehat{\rho}(t, \omega)-\rho(t, \omega) \widetilde{\rho}(t, \omega)|>0 .
$$

This condition ensures that system $(17)$ defines a unique $\theta \in \mathcal{T}$ for any $\mathcal{F}_{t}$-adapted process $\varrho$ such that $\operatorname{ess}_{\sup } \int_{0}^{T} \varrho(t)^{2} d t<+\infty$.

Example 2 For $\theta$ from Theorem 7 with $\varrho \equiv 0$, the process $(S(t), B(t))$ evolves as

$$
\begin{aligned}
& d S(t)=S(t)\left([a(t)-\widetilde{a}(t)] d t+\sigma(t) d W_{1 \theta}(t)+\widehat{\sigma}(t) d W_{2 \theta}(t)\right), \\
& \left.d B(t)=B(t)\left(\alpha(t) d t+\rho(t) d W_{1 \theta}(t)\right)+\widehat{\rho}(t) d W_{2 \theta}(t)\right) .
\end{aligned}
$$

In this case, the equation for $B$ has the same coefficients as the equation for $B(t)$ under $\mathbf{P}$, with replacement of $W(t)$ by $W_{\theta}(t)$. Respectively, the distribution of $B(t)$ under $\mathbf{P}_{\theta}$ and under the historical measure $\mathbf{P}$ is the same if the coefficients $\alpha(t), \rho(t)$, and $\widehat{\rho}(t)$, are non-random. In addition, if $\widehat{\sigma} \equiv 0$ then the choice $\varrho \equiv 0$ ensures that $\theta_{1}=\widehat{a} / \sigma$.

Example 3 For $\theta$ from Theorem 7 with $\varrho=-\widetilde{a}$, the evolution of $S$ under $\mathbf{P}_{\theta}$ is described by an Itô equation with the same coefficients as the equation for $S(t)$ under $\mathbf{P}$, with replacement of $W(t)$ by $W_{\theta}(t)$. In this case, the distribution of $S(t)$ under $\mathbf{P}_{\theta}$ and under the historical measure $\mathbf{P}$ is the same if the coefficients $a(t), \sigma(t)$, and $\widehat{\sigma}(t)$, are non-random.

Example 4 Let $k \in(0,1)$ and $r_{B} \in \mathbf{R}$ be given. Let us calculate $\theta$ from (17) with $\varrho=$ $k\left(\alpha-r_{B}\right)$ i.e.,

$$
\begin{aligned}
& \widetilde{\sigma} \theta_{1}+\widetilde{\rho} \theta_{2}=\widetilde{a}, \\
& \rho \theta_{1}+\widehat{\rho} \theta_{2}=k\left(\alpha-r_{B}\right) .
\end{aligned}
$$

By (18), this leads to the equation

$$
\left.d B(t)=B(t)\left(\left[k r_{B}+(1-k) \alpha\right] d t+\rho d W_{1 \theta}(t)\right)+\widehat{\rho} d W_{2 \theta}(t)\right),
$$

i.e., the appreciation rate coefficient for $B$ under $\mathbf{P}_{\theta}$ is $k r_{B}+(1-k) \alpha$. Therefore, there exists a choice of $\theta$ that ensures that the appreciation rate for $B$ under $\mathbf{P}_{\theta}$ can be arbitrarily close to $r_{B}$. This can be achieved with selection of $k$ close to 1 in (20). 
In is also possible to consider a modification of this approach with $r_{B}$ depending on $\theta$. For example, an important example considered below is where $r_{B}=\alpha-r_{\theta}$, where

$$
r_{\theta}=\alpha-\rho^{2}-\widehat{\rho}^{2}-\rho \theta_{1}-\widehat{\rho} \theta_{2} .
$$

(see (32) below). This gives equations

$$
\begin{aligned}
& \tilde{\sigma} \theta_{1}+\widetilde{\rho} \theta_{2}=\widetilde{a}, \\
& \rho \theta_{1}+\widehat{\rho} \theta_{2}=\frac{k}{1-k}\left(\rho^{2}+\hat{\rho}^{2}\right) .
\end{aligned}
$$

In particular, this $r_{B}$ can be selected as the expected average risk-free rate associated with the zero-coupon bond under the measure $\mathbf{P}_{\theta}$, as is described in the next section.

Example 5 An important example of the selection of $\theta$ is

$$
\theta(t)=\widetilde{a}(t) V(t) /|V(t)|^{2}
$$

The following theorem shows that this corresponds to the choice of $\theta$ with the minimal norm.

Theorem 8 Let $\theta(t)$ be defined by (22). Then, for every $t, \omega$, the value of $|\theta(t, \omega)|$ is minimal among all $\theta \in \mathcal{T}$. In addition, if $\xi=c_{\theta}+\int_{0}^{T} \gamma_{\theta}(t) d \widetilde{S}(t)+R_{\theta}$ for some $R_{\theta} \in \mathcal{X}_{\theta}^{\top}$ and $\gamma_{\theta}$ is an adapted process such that $\gamma_{\theta} \sigma \in \mathcal{H}_{\theta}$, then $\mathbf{E}\left(R_{\theta} \mathcal{M}(T)\right)=0$, where $\mathcal{M}(T)=$ $\int_{0}^{T} \gamma_{\theta}(t) \widetilde{S}(t) V(t)^{\top} d W(t)$ represents the "martingale" part of the integral

$$
\int_{0}^{T} \gamma_{\theta}(t) d \widetilde{S}(t)=\int_{0}^{T} \gamma_{\theta}(t) \widetilde{S}(t) \widehat{a}(t) d t+\mathcal{M}(T)
$$

The selection of $\theta$ described in Theorem 8 ensures that the corresponding self-financing strategy with the quantity of shares $\gamma(t)$ is a so-called locally risk minimizing strategy; see, e.g., Föllmer and Sondermann (1986), Biagini and Pratelli (1999).

Let us reconsider Example 1 (iv). We will be using the measure $\widehat{\mathbf{P}}$ and the processes $q(t)$, $z(t)$, and $\widehat{z}(t)$ defined in this example.

Set $\mathcal{V}(t)=\widehat{V}(t)-k(t) V$, where

$$
k(t)=\widehat{V}(t)^{\top} V(t) /|V(t)|^{2} .
$$

Clearly, we have that $\mathcal{V}(t)^{\top} V(t)=0$.

Further, there exists a one-dimensional Wiener process $z_{1}(t)$ such that $\int_{0}^{t} \mathcal{V}(s)^{\top} d W(s)=$ $\int_{0}^{t}|\mathcal{V}(s)| d z_{1}(s)$ and

$$
\begin{aligned}
d B(t) & =B(t)\left(\alpha(t) d t+k(t) V(t)^{\top} d W(t)+\mathcal{V}(t)^{\top} d W(t)\right) \\
& =B(t)\left(\alpha(t) d t+k(t)|V(t)| d z(t)+|\mathcal{V}(t)| d z_{1}(t)\right)
\end{aligned}
$$


For $q(t)=\widetilde{a}(t) /|V(t)|$, we have

$$
d B(t)=B(t)\left(\alpha(t) d t+k(t)|V(t)|(d \widehat{z}(t)-q(t) d t)+|\mathcal{V}(t)| d z_{1}(t)\right) .
$$

On the other hand,

$$
d B(t)=B(t)\left(\alpha(t) d t+\widehat{V}(t)^{\top} d W(t)\right)=B(t)\left(\alpha(t) d t+\widehat{V}(t)^{\top}\left(d W_{\theta}(t)-\theta(t) d t\right) .\right.
$$

This means that, in our notation, $\widehat{\mathbf{P}}=\mathbf{P}_{\theta}$, where $\theta \in \mathcal{T}$ is such that

$$
k(t) q(t)|V(t)|=k(t) \widetilde{a}(t)=\widehat{V}(t)^{\top} \theta(t) .
$$

The only $\theta \in \mathcal{T}$ satisfying this is $\theta(t)=\widetilde{a}(t) V(t) /|V(t)|^{2}$ from Theorem 8 .

\section{Risk-free bonds and choice of $\theta$ implied from the bond prices}

In this section, we suggest one more approach to selection of equivalent martingale measures. First, it can be noted that the market described above does not include a risk free asset since both processes $S(t)$ and $B(t)$ are random. However, one can augment this market with auxiliary tradable risk-free assets representing zero-coupon bonds constructed as some options with the payoff $\$ 1$ at time $T$. We represent the price of these bonds as a function of $\theta$. On the next step, we inverse this pricing formula and represent $\theta$ via observed market bond prices. This gives a way of selection of implied parameter $\theta_{\text {implied }}$ consistent with the observed bond prices. It follows the classical approach to the so-called implied volatility where the Black-Scholes formula is reversed.

\subsection{The implied market price of risk for complete market}

Let us assume first that

$$
\widehat{\sigma}(t) \equiv \widehat{\rho}(t) \equiv 0
$$

In this case, by the definition of $\mathcal{F}_{t}$, we have that $\mathcal{F}_{t}=\mathcal{F}_{t}^{w}$, and the assumptions of Theorem 2 are satisfied. In this case, $\widetilde{\rho} \equiv 0$, and (17) implies that $\varrho \equiv \rho \theta_{1}$ and that $\theta_{1}(t)$ is uniquely defined as $\theta_{1}(t)=\widetilde{\sigma}(t)^{-1} \widetilde{a}(t)$. The process $\theta_{1}$ is called the marked price of risk process in this case.

Under these assumptions, the equation for $B$ in (18) can rewritten as

$$
\left.d B(t)=B(t)\left(\left[\alpha(t)-\rho(t) \theta_{1}(t)\right] d t+\rho(t) d W_{1 \theta}(t)\right)\right) .
$$


Lemma 3 if (23) holds, then the claim $\xi \equiv \$ 1$ is replicable in the following sense: for any $t \in[0, T)$, there exists an $\mathcal{F}_{t}$-adapted process $\gamma(t)$ such that $\mathbf{E}_{\theta} \int_{0}^{T} \gamma(t)^{2} \widetilde{S}(t)^{2} d t<+\infty$ and

$$
B(T)^{-1}=\mathbf{E}_{\theta} B(T)^{-1}+\int_{0}^{T} \gamma(t) d \widetilde{S}(t) .
$$

Remind that, by the definition of $\mathcal{F}_{t}$, the processes $a(t), \sigma(t), \alpha(t)$, and $\rho(t)$, are $\mathcal{F}_{t}^{w}$ adapted if (23) holds.

Under the assumptions of Lemma 3 , the value $\mathbf{E}_{\theta} B(T)^{-1}$ represents the price at time $t=0$ of a zero-coupon bond with the payoff $\$ 1$ at the maturity time $T$. The value $\widetilde{X}(t)=$ $\mathbf{E}_{\theta}\left\{B(T)^{-1} \mid \mathcal{F}_{t}\right\}$ represents the discounted wealth for the hedging (replicating) strategy, as well as the value

$$
P(t, T)=B(t) \widetilde{X}(t)=B(t) \mathbf{E}_{\theta}\left\{B(T)^{-1} \mid \mathcal{F}_{t}\right\}
$$

represents the total wealth for the replicating strategy and the price at time $t$ of a zero-coupon bond with the payoff $\$ 1$ at the maturity time $T$.

Let us discuss some consequences of these statements.

Lemma 3 implies that the value

$$
r(t) \triangleq-(T-t)^{-1} \log P(t, T)=-(T-t)^{-1} \log \left(B(t) \mathbf{E}_{\theta}\left\{B(T)^{-1} \mid \mathcal{F}_{t}\right\}\right)
$$

represents the so-called yield to maturity, or the expected average risk-free rate associated with the zero-coupon bond, meaning that the price at time $t$ of a zero-coupon bond with the payoff $\$ 1$ at the maturity time $T$ is

$$
P(t, T)=\exp (-r(t)(T-t))
$$

If the processes $a(t), \sigma(t), \alpha(t), \rho(t), \widehat{\rho}(t), \theta(t)$ are constant and the assumptions of Lemma 3 are satisfied, then

$$
B(T)^{-1}=B(t)^{-1} \exp \left(\left(-\alpha+\frac{\rho^{2}}{2}+\rho \theta_{1}\right)[T-t]-\rho\left(W_{1 \theta}(T)-W_{1 \theta}(t)\right)\right) .
$$

In this case, a direct calculation of (25) gives

$$
P(t, T)=B(t) \mathbf{E}_{\theta}\left\{B(T)^{-1} \mid \mathcal{F}_{t}\right\}=\exp \left[-(T-t)\left(\alpha-\rho^{2}-\rho \theta_{1}\right)\right]
$$

and

$$
-(T-t)^{-1} \log P(t, T)=\alpha-\rho^{2}-\rho \theta_{1} .
$$


Since $\theta_{1}=\widetilde{a} / \widetilde{\sigma}$, it gives that $-(T-t)^{-1} \log P(t, T)=\alpha-\rho^{2}-\rho \widetilde{a} / \widetilde{\sigma}$. Hence

$$
r \triangleq \alpha-\rho^{2}-\rho \widetilde{a} / \widetilde{\sigma}
$$

can be interpreted as the "true" risk-free rate for this market. It can be seen that $r$ is close to $\alpha$ if $\rho$ is small. If $\rho=0$ then $r=\alpha$.

Let us investigate the situation where $\theta_{1}$ is unknown. Let us consider a scenario where the real market price $P_{\text {market }}(0, T)$ of the zero-coupon bond with the payoff $\$ 1$ at the maturity time $T$ is observable at time $t=0$. In this case, the corresponding value (26) $r_{\text {market }}=$ $-T^{-1} \log P_{\text {market }}(0, T)$ can be calculated. By the assumptions on the coefficients, (23) implies that $\rho \neq 0$. In this case, we can reverse pricing formula (25) (or reverse (29)) and calculate implied $\theta_{1, \text { implied }}$ from (29) as

$$
\theta_{1, \text { implied }}=\left(\alpha-\rho^{2}-r_{\text {market }}\right) / \rho .
$$

In this case, equation (1) can be rewritten as

$$
d B(t)=B(t)\left(\left[r_{\text {market }}+\rho^{2}\right] d t+\rho d W_{1 \theta}(t)\right),
$$

where $W_{1 \theta}(t)=w(t)+\int_{0}^{t} \theta_{1, \text { implied }}(s) d s$.

It particular, it follows that a choice of $\rho$ for a market model with given $\alpha$ is consistent with the observed bond prices if $\left(\alpha-r_{\text {market }}\right) / \rho$ is bounded as $\rho \rightarrow 0$. This leads to the following heuristic rule: if the observed bond market price is such that $\alpha-r_{\text {market }}$ is large, then one should assume a sufficiently large $\rho$, to avoid overestimation of the market price of risk.

Representation (30) follows the classical approach to the so-called implied volatility where the Black-Scholes formula is reversed. Representation of the market price of risk process $\theta_{1}$ implied form observed bond prices as described above could be a useful addition to the existing methods. Further development of this approach is presented in Section 5.1 below.

Remark 4 For the bond pricing model with constant coefficients described above, the choice of $\left(\theta_{1}, r\right)$ is independent on $T$. It follows that a single market price $P_{\text {market }}(0, T)$ of a zero coupon bond for one given maturity time $T$ defines uniquely the prices of similar bonds for all other maturity times $\bar{T} \neq T$ given that these prices are defined by (25). This is caused by the fact that this formula has to be applied with the same $\theta_{1}$ leading to the same $r$ in (27).

\section{Implied $\theta$ from observed bond prices for the incomplete market}

We suggested above a method of calculation of the implied market price of risk $\theta_{1, \text { implied }}$ from observed bond prices for a case of complete market with non-zero $\rho$ and $\widehat{\rho}$. For this, we 
established that the value (25) represents the price at time $t$ of a zero-coupon bond with the payoff $\$ 1$ at the maturity time $T$ for a case where the claim $\$ 1$ is replicable. This indicates that it could be reasonable to accept (25) as the price of this bond for a more general case for an incomplete market with a non-replicable claim $\$ 1$ as well (i.e., with non-zero $\rho$ and $\widehat{\rho}$ ).

Up to the end of this section, we assume a market model with non-zero $\rho$ and $\widehat{\rho}$, where the price a zero-coupon bond with the payoff $\$ 1$ at the maturity time $T$ is defined by (25) similarly to the case of the complete market, regardless of replicability of the corresponding contingent claim.

This leads to an one more important example of selection of $\varrho$ and of the corresponding $\theta$ defined by (17) that supplements choices described in Section 4.

Assume that the processes $a(t), \sigma(t), \alpha(t), \rho(t), \widehat{\rho}(t), \theta(t)$ are constant, and that $r(t)$ is defined by (26). This means that $r(t)$ represents again the expected average risk-free rate associated with the zero-coupon bond.

It follows from the Itô formula that

$$
\begin{aligned}
B(T)^{-1}= & B(t)^{-1} \exp \left(\left(-\alpha+\frac{\rho^{2}}{2}+\frac{\widehat{\rho}^{2}}{2}+\rho \theta_{1}+\widehat{\rho} \theta_{2}\right)(T-t)\right. \\
& \left.-\rho\left(W_{1 \theta}(T)-W_{1 \theta}(t)\right)-\widehat{\rho}\left(W_{2 \theta}(T)-W_{2 \theta}(t)\right)\right)
\end{aligned}
$$

In this case, a direct calculation of (25) gives

$$
P(t, T)=B(t) \mathbf{E}_{\theta}\left\{B(T)^{-1} \mid \mathcal{F}_{t}\right\}=e^{-(T-t)\left(\alpha-\rho^{2}-\widehat{\rho}^{2}-\rho \theta_{1}-\widehat{\rho} \theta_{2}\right)}
$$

and

$$
-(T-t)^{-1} \log P(t, T)=\alpha-\rho^{2}-\widehat{\rho}^{2}-\rho \theta_{1}-\widehat{\rho} \theta_{2}
$$

Then (26) imply that $r(t)$ can be found explicitly; it does not depend on $t$ and $T$ and depend on $\theta$ such that $r(t) \equiv r_{\theta}$, where

$$
r_{\theta}=\alpha-\rho^{2}-\widehat{\rho}^{2}-\rho \theta_{1}-\widehat{\rho} \theta_{2}
$$

Using (17), equation (32) can be rewritten as

$$
\varrho=\rho \theta_{1}+\widehat{\rho} \theta_{2}=\alpha-r_{\theta}-\rho^{2}-\widehat{\rho}^{2} .
$$

Following the approach from Section 5, consider now a scenario where the real market price $P_{\text {market }}(0, T)$ of a zero-coupon bond with the payoff $\$ 1$ at the maturity time $T$ is observed from the market statistics at time $t=0$, and the corresponding value $(25)$ 
$r_{\text {market }}=-T^{-1} \log P_{\text {market }}(0, T)$ is calculated. We can reverse pricing formula (25) and calculate implied $\varrho$ as

$$
\varrho_{\text {implied }}=\alpha-r_{\text {market }}-\rho^{2}-\widehat{\rho}^{2}
$$

and find implied $\theta=\theta_{\text {implied }}=\left(\theta_{\text {implied }, 1}, \theta_{\text {implied }, 2}\right)^{\top}$ as solution of corresponding system (17), i.e.

$$
\begin{aligned}
\widetilde{\sigma} \theta_{\text {implied }, 1}+\widetilde{\rho} \theta_{\text {implied }, 2} & =\widetilde{a}, \\
\rho \theta_{\text {implied }, 1}+\widehat{\rho} \theta_{\text {implied }, 2} & =\varrho_{\text {implied }} .
\end{aligned}
$$

This would follow again the classical approach to the so-called implied volatility where the Black-Scholes formula is reversed.

In particular, equation (18) for this $\varrho=\varrho_{\text {implied }}$ gives that

$$
\left.d B(t)=B(t)\left(\left[r_{\text {market }}+\rho^{2}+\widehat{\rho}^{2}\right] d t+\rho d W_{1 \theta}(t)\right)+\widehat{\rho} d W_{2 \theta}(t)\right),
$$

where $\theta=\theta_{\text {implied }}$.

This model has the same feature as described in Remark 4 for a special model. In particular, the choice of $\theta$ is independent on $T$, and a single market price $P_{\text {market }}(0, T)$ of a zero coupon bond for one given maturity time $T$ defines uniquely the prices defined by (25) for similar bonds for all other maturity times $\bar{T} \neq T$, since this formula has to be applied with the same $\theta$. For models with time variable coefficients of equations for $B$ and $S$, the same approach gives a time dependent solution $\theta(t)$ of (17), and the value $r$ defined by (29) for a maturity time $\bar{T}$ depends on $\bar{T}$.

\section{Markov case}

The values for the prices, errors, and hedging strategies obtained above were expressed via integrands which existence is ensured by the Martingale Representation Theorem. In this section, we suggests some representations via solutions of deterministic PDEs which could be more convenient.

Assume that $\theta \in \mathcal{T}$ is given.

We will be using the processes $\mathrm{s}(t)=\log \widetilde{S}(t)$ and $\mathrm{b}(t)=\log B(t)$. By Itô formula, it follows that

$$
\begin{aligned}
d \mathrm{~s}(t) & =\left(\widetilde{a}-\widetilde{\sigma}^{2} / 2-\widetilde{\rho}^{2} / 2\right) d t+\widetilde{\sigma} d w(t)+\widetilde{\rho} d \widehat{w}(t) \\
& =\left(-\widetilde{\sigma}^{2} / 2-\widetilde{\rho}^{2} / 2\right) d t+V(t)^{\top} d W_{\theta}(t),
\end{aligned}
$$


where $V^{\top}=(\widetilde{\sigma}, \widetilde{\rho})^{\top}$, and

$$
\begin{aligned}
d \mathrm{~b}(t) & =\left(\alpha-\rho^{2} / 2-\widehat{\rho}^{2} / 2\right) d t+\rho d w(t)+\widehat{\rho} d \widehat{w}(t) \\
& =\left(\alpha-\rho^{2} / 2-\widehat{\rho}^{2} / 2\right) d t+\widehat{V}(t)^{\top} d W_{\theta}(t),
\end{aligned}
$$

where $\widehat{V}^{\top}=(\rho, \widehat{\rho})^{\top}$.

Up to the end of this section, we assume that there exists a measurable function $\Theta$ : $\mathbf{R}^{2} \times[0, T] \rightarrow \mathbf{R}^{8}$ such that

$$
\left(\widetilde{a}(t), \widetilde{\sigma}(t), \widetilde{\rho}(t), \alpha(t), \rho(t), \widehat{\rho}(t), \theta_{1}(t), \theta_{2}(t)\right)^{\top}=\Theta(\mathrm{s}(t), \mathrm{b}(t), t) .
$$

To simplify notation, we will describe it as the following: we assume that the processes $\widetilde{a}(t)$, $\widetilde{\sigma}(t), \widetilde{\rho}(t), \alpha(t), \rho(t), \widehat{\rho}(t), \theta(t), V(t), \widehat{V}(t)$ (defined on $[0, T] \times \Omega$ ) are replaced by the processes $\widetilde{a}(\mathrm{~s}(t), \mathrm{b}(t), t), \widetilde{\sigma}(\mathrm{s}(t), \mathrm{b}(t), t), \widetilde{\rho}(\mathrm{s}(t), \mathrm{b}(t), t), \alpha(\mathrm{s}(t), \mathrm{b}(t), t), \rho(\mathrm{s}(t), \mathrm{b}(t), t), \widehat{\rho}(\mathrm{s}(t), \mathrm{b}(t), t), \theta(\mathrm{s}(t), \mathrm{b}(t), t)$, $V(\mathrm{~s}(t), \mathrm{b}(t), t)$, and $\widehat{V}(\mathrm{~s}(t), \mathrm{b}(t), t)$, respectively, for some measurable functions $\widetilde{a}(s, b, t), \widetilde{\sigma}(s, b, t)$, $\widetilde{\rho}(s, b, t), \alpha(s, b, t), \rho(s, b, t), \widehat{\rho}(s, b, t), \theta(s, b, t), V(s, b, t), \widehat{V}(s, b, t)$, defined on $\mathbf{R}^{2} \times[0, T]$.

Let $H=H_{\theta}=H_{\theta}(s, b, t)$ be the solution of the following Cauchy problem for a linear parabolic equation in $\mathbf{R}^{2} \times[0, T]$

$$
\begin{aligned}
& H_{t}^{\prime}+H_{s}^{\prime}\left(-\widetilde{\sigma}^{2} / 2-\widetilde{\rho}^{2} / 2\right)+H_{b}^{\prime}\left(\alpha-\rho^{2} / 2-\widehat{\rho}^{2} / 2\right)+\mathcal{L} H=0, \\
& H(s, b, T)=F\left(e^{s}, e^{b}\right), \quad(s, b) \in \mathbf{R}^{2} .
\end{aligned}
$$

Here

$$
\mathcal{L} H=\frac{1}{2}\left(\begin{array}{c}
\widetilde{\sigma} \\
\rho
\end{array}\right)^{\top} H^{\prime \prime}\left(\begin{array}{c}
\widetilde{\sigma} \\
\rho
\end{array}\right)+\frac{1}{2}\left(\begin{array}{c}
\widetilde{\rho} \\
\widehat{\rho}
\end{array}\right)^{\top} H^{\prime \prime}\left(\begin{array}{c}
\widetilde{\rho} \\
\widehat{\rho}
\end{array}\right), \quad H^{\prime \prime}=\left(\begin{array}{cc}
H_{s s}^{\prime \prime} & H_{s b}^{\prime \prime} \\
H_{b s}^{\prime \prime} & H_{b b}^{\prime \prime}
\end{array}\right) .
$$

In this section, we assume that there exists a generalized solution $H(s, b, t)$ of Cauchy problem (37) such that its gradient with respect to $(s, b)$ is bounded.

Under these assumptions, consider the pricing problem for the claim $B(T) \xi$, where $\xi=$ $F(\widetilde{S}(T), B(T))$ for some measurable function $F:(0,+\infty)^{2} \rightarrow \mathbf{R}$ such that $\mathbf{E}_{\theta} \xi^{2}<+\infty$ for some $\theta \in \mathcal{T}$.

Proposition 3 The price in (11) can be represented as

$$
c_{\theta}=\mathbf{E}_{\theta} \xi=H(\mathrm{~s}(0), \mathrm{b}(0), 0)=H(\log \widetilde{S}(0), \log B(0), 0) .
$$

Furthermore, the hedging strategy in (14) can be represented as

$$
\gamma(t)=f_{\theta}(\mathrm{s}(t), \mathrm{b}(t), t) e^{-\mathrm{s} t}=f_{\theta}(\log \widetilde{S}(t), \log B(t), t) \widetilde{S}(t)^{-1},
$$


where

$$
f_{\theta}(s, b, t)=H_{s}^{\prime}(\mathrm{s}(t), \mathrm{b}(t), t)+H_{b}^{\prime}(\mathrm{s}(t), \mathrm{b}(t), t) \frac{\widetilde{\sigma}(s, b, t) \rho(s, b, t)+\widetilde{\rho}(s, b, t) \widehat{\rho}(s, b, t)}{\widetilde{\sigma}(s, b, t)^{2}+\widetilde{\rho}(s, b, t)^{2}} .
$$

Further, let us consider the problem of estimation of $\mathbf{E} R_{\theta}^{2}$ for the discounted hedging error $R_{\theta}$. This error is represented in (11)-(14) as

$$
R_{\theta}=\mathbf{E} \int_{0}^{T} \eta_{\theta}(t)^{\top} d W_{\theta}(t), \quad \eta_{\theta}(t)=U_{\theta}(t)-\nu_{\theta}(t) V(t) .
$$

Consider a function $g_{\theta}=g_{\theta}(s, b, t): \mathbf{R}^{2} \times[0, T] \rightarrow \mathbf{R}$ defined as $g_{\theta}(s, b, t)=H_{s}^{\prime}(s, b, t)\left(\begin{array}{c}\tilde{\sigma}(s, b, t) \\ \widetilde{\rho}(s, b, t)\end{array}\right)+H_{b}^{\prime}(s, b, t)\left(\begin{array}{c}\rho(s, b, t) \\ \widehat{\rho}(s, b, t)\end{array}\right)-f_{\theta}(s, b, t)\left(\begin{array}{c}\tilde{\sigma}(s, b, t) \\ \widetilde{\rho}(s, b, t)\end{array}\right)$.

Let $J=J(y, s, b, t)$ be the solution of the following Cauchy problem for a linear parabolic equation in $\mathbf{R}^{3} \times[0, T]$

$$
\begin{aligned}
& J_{t}^{\prime}+J_{y}^{\prime} g_{\theta}^{\top} \theta+J_{s}^{\prime}\left(\widetilde{a}-\widetilde{\sigma}^{2} / 2-\widetilde{\rho}^{2} / 2\right)+J_{b}^{\prime}\left(r-\rho^{2} / 2-\widehat{\rho}^{2} / 2\right)+\mathcal{D} J \\
& J(y, s, b, T)=y^{2}, \quad(y, s, b) \in \mathbf{R}^{3} .
\end{aligned}
$$

Here $g_{\theta}=\left(g_{\theta, 1}, g_{\theta, 2}\right)^{\top}$,

$\mathcal{D} J=\frac{1}{2}\left(\begin{array}{c}g_{\theta, 1} \\ \widetilde{\sigma} \\ \rho\end{array}\right)^{\top} J^{\prime \prime}\left(\begin{array}{c}g_{\theta, 1} \\ \widetilde{\sigma} \\ \rho\end{array}\right)+\frac{1}{2}\left(\begin{array}{c}g_{\theta, 2} \\ \widetilde{\rho} \\ \widehat{\rho}\end{array}\right)^{\top} J^{\prime \prime}\left(\begin{array}{c}g_{\theta, 2} \\ \widetilde{\rho} \\ \widehat{\rho}\end{array}\right), \quad J^{\prime \prime}=\left(\begin{array}{ccc}J_{x x}^{\prime \prime} & J_{x y}^{\prime \prime} & J_{x z}^{\prime \prime} \\ J_{y x}^{\prime \prime} & J_{y y}^{\prime \prime} & J_{y z}^{\prime \prime} \\ J_{z x}^{\prime \prime} & J_{z y}^{\prime \prime} & J_{z z}^{\prime \prime}\end{array}\right)$.

We assume that there exists a generalized solution $J(y, s, b, t)$ of Cauchy problem (38) such that its gradient with respect to $(y, s, b)$ is bounded.

Proposition 4 We have that

$$
\mathbf{E} R_{\theta}^{2}=J(0, \log \mathrm{s}(0), \mathrm{b}(0), 0)=J(0, \log \widetilde{S}(0), \log B(0), 0) .
$$

\section{Conclusions}

We revisited the problem of pricing of stock options for the case of the market model with a stochastic numéraire, with emphasize on the impact of the non-uniqueness of equivalent martingale measures and the presence of non-replicable claims. Some possible economically justified choices of equivalent martingale measures are suggested, including a measure that correspond to a consensus about the future numéraire process, a measure that ensures local 
risk minimizing hedging strategy, and an implied measure that takes into account observed zero coupon bond prices. This last measure is especially interesting, since it leads to a formula for the implied market price of risk.

It could be interesting to consider optimal selection of the equivalent martingale measure in the spirit of the mean-variance hedging. It also could be interesting to develop a comprehensive bond pricing model that is based on stochastic numéraire with time dependent coefficients, and investigate dependence of the price on the maturity time. We leave it for future research.

\section{Appendix: Proofs}

Proof of Lemma 1 is straightforward and based on the application of Itô's formula. In fact, Lemma 1 represents a special case of Proposition 1 from Geman et al. (1995).

Proof of Lemma 2 follows immediately from equation (9) and from the fact that $d \widetilde{S}(t)=$ $\widetilde{S}(t) V(t)^{\top} d W_{\theta}(t)$.

Proof of Proposition 2. By Lemma 1 and 2, the set $\mathcal{X}_{\theta}$ contains random variables

$$
\int_{0}^{T} \gamma(t) d \widetilde{S}(t)=\int_{0}^{T} \gamma(t) \widetilde{S}(t) V(t)^{\top} d W_{\theta}(t)
$$

where $\gamma \in \mathcal{H}_{\theta}$ and where $W_{\theta}$ is defined by (6).

For any $\zeta \in \mathcal{X}_{\theta}^{\perp}$, there exists $U(t)=\left(U_{1}(t), U_{2}(t)\right)^{\top} \in \mathcal{Y}_{\theta}$ such that

$$
\zeta=\int_{0}^{T} U(t)^{\top} d W_{\theta}(t)
$$

Let us show that if $\zeta \in \mathcal{X}_{\theta}^{\perp}$ then $U(t)^{\top} V(t)=0$. For this $\zeta$, we have that

$$
\mathbf{E}_{\theta} \zeta \int_{0}^{T} \gamma(t) d \widetilde{S}(t)=\mathbf{E}_{\theta} \int_{0}^{T} \gamma(t) \widetilde{S}(t) V(t)^{\top} U(t) d t=0 \quad \forall \gamma \in \mathcal{H}_{\theta}
$$

Hence $\widetilde{S}(t) V(t)^{\top} U(t)=0$ a.e. Hence $V(t)^{\top} U(t)=0$ a.e.

To show that the set $\mathcal{X}_{\theta}^{\perp}$ contains non-zero elements, it suffices to take $U_{1}(t)=\psi(t) \widehat{\rho}(t)$ and $U_{2}(t)=\psi(t) \widetilde{\sigma}(t)$, with an arbitrary $\psi \in \mathcal{Y}_{\theta}$, i.e.,

$$
\zeta=\int_{0}^{T} \psi(t)\left[\widehat{\rho}(t) d W_{\theta 1}(t)+\widetilde{\sigma}(t) d W_{\theta 2}(t)\right]
$$

This completes the proof.

Proof of Theorem 1. Under the assumptions, $d \widetilde{S}(t)=\widetilde{S}(t)|V(t)| d z_{\theta}(t)$, where $z_{\theta}(t)$ is a one-dimensional Wiener process such that $\int_{0}^{t} V(s)^{\top} d W_{\theta}(s)=\int_{0}^{t}|V(s)| d z_{\theta}(s)$. Hence the 
filtration $\left\{\mathcal{F}_{t}^{z_{\theta}}\right\}_{t \geq o}$ generated by $z_{\theta}(t)$ is such that $\mathcal{F}_{T}^{z_{\theta}}=\mathcal{F}_{T}^{\widetilde{S}}$. Hence any $\xi \in L_{2}\left(\Omega, \mathcal{F}_{T}^{\widetilde{S}}, \mathbf{P}_{\theta}\right)$ belongs to $L_{2}\left(\Omega, \mathcal{F}_{T}^{z_{\theta}}, \mathbf{P}_{\theta}\right)$. By the Martingale Representation Theorem, it follows that there exists an $\mathcal{F}_{t}^{z_{\theta}}$-adapted process $u_{\theta}(t)$ such that $\mathbf{E}_{\theta} \int_{0}^{T} u_{\theta}(t)^{2} d t<+\infty$ and $\xi=\mathbf{E}_{\theta} \xi+$ $\int_{0}^{T} u_{\theta}(t) d z_{\theta}(t)$. It suffices to select $\gamma(t)=u_{\theta}(t) \widetilde{S}(t)^{-1}$. This completes the proof of Theorem 1 .

Proof of Theorem 2 follows immediately from the Martingale Representation Theorem applied on the probability space $\left(\Omega, \mathcal{F}_{T}^{w}, \mathbf{P}_{\theta}\right)$.

Proof of Corollary 1. Let the initial wealth $c_{\theta i}=X^{(i)}(0)$ and the strategy $\left(\beta^{(i)}(\cdot), \gamma^{(i)}(\cdot)\right)$ be such that $\widetilde{X}^{(i)}(T)=\xi$ a.s. for the corresponding discounted wealth $X^{(i)}(t), i=1,2$.

Set

$$
g(t) \triangleq \gamma^{(1)}(t)-\gamma^{(2)}(t), \quad Y(t) \triangleq \widetilde{X}^{(1)}(t)-\widetilde{X}^{(2)}(t) .
$$

We have that $Y(T)=0$ a.s.. Hence

$$
Y(T)=Y(0)+\int_{0}^{T} g(t) d \widetilde{S}(t)=0 .
$$

For $K>0$, consider first exit times $T_{K}=T \wedge \inf \left\{t: \int_{0}^{t}\left(\left|\gamma^{(1)}(s)\right|+\left|\gamma^{(2)}(s)\right|^{2} d s \geq K\right\}\right.$; they are Markov times with respect to $\left\{\mathcal{F}_{t}\right\}_{t \geq 0} t$. We have that

$$
Y\left(T_{K}\right)=Y(0)+\int_{0}^{T_{K}} g(t) d \widetilde{S}(t)=\mathbf{E}_{\theta i}\left\{Y(T) \mid \mathcal{F}_{T_{K}}\right\}=0, \quad i=1,2 .
$$

Hence

$$
0=Y(0)^{2}+\mathbf{E}_{\theta_{i}} \int_{0}^{T_{K}} g(s)^{2} \widetilde{S}(s)^{2}|V(s)|^{2} d t=0 .
$$

It follows that $Y(0)=0$, and $\left.g(t)\right|_{\left[0, T_{K}\right]}=0$ for any $K>0$. In addition, $T_{K} \rightarrow T$ a.s. as $T_{K} \rightarrow+\infty$. Hence $g=0$. This completes the proof of Corollary 1 .

Proof of Theorem 3. By (16), for any $K \in \mathbf{R}$, there exists $\theta=\theta_{K} \in \mathcal{T}$ such that

$$
\begin{aligned}
& \theta_{1} \widetilde{\sigma}+\theta_{2} \widetilde{\rho}=\widetilde{a}, \\
& \theta_{1} \rho+\theta_{2} \widehat{\rho}=\widehat{V}(t)^{\top} \theta(t)=K-\alpha+\rho^{2}+\widehat{\rho}^{2} .
\end{aligned}
$$

By Girsanov's Theorem, $W_{\theta}(t)=W(t)+\int_{0}^{t} \theta(s) d s$ is a standard Wiener process in $\mathbf{R}^{2}$ under $\mathbf{P}_{\theta}$. We have that $d \widetilde{S}(t)=\widetilde{S}(t) V(t)^{\top} d W_{\theta}(t)$ and

$$
\begin{aligned}
d B(t)^{-1} & =B(t)^{-1}\left(\left[-\alpha+\rho^{2}+\widehat{\rho}^{2}\right] d t-\rho d w(t)-\widehat{\rho} d \widehat{w}(t)\right) \\
& =B(t)^{-1}\left(\left[-\alpha+\rho^{2}+\widehat{\rho}^{2}\right] d t-\widehat{V}(t)^{\top} d W(t)\right) \\
& =B(t)^{-1}\left(\left[-\alpha+\rho^{2}+\widehat{\rho}^{2}\right] d t-\widehat{V}(t)^{\top} \theta(t) d t+\widehat{V}(t)^{\top} d W_{\theta}(t)\right) \\
& =B(t)^{-1}\left(\left[-\alpha+\rho^{2}+\widehat{\rho}^{2}\right] d t-\left(K-\alpha+\rho^{2}+\widehat{\rho}^{2}\right) d t+\widehat{V}(t)^{\top} d W_{\theta}(t)\right) \\
& =B(t)^{-1}\left(-K d t+\widehat{V}(t)^{\top} d W_{\theta}(t)\right) .
\end{aligned}
$$


Let $\widehat{B}(t)^{-1}=e^{K t} B(t)^{-1}$. We have that

$$
d \widehat{B}(t)^{-1}=\widehat{B}(t)^{-1} \widehat{V}(t)^{\top} d W_{\theta}(t) .
$$

It follows that $\widehat{B}(t)^{-1}$ is a martingale under $\mathbf{P}_{\theta}$.

Let us prove statement (i) of Theorem 3 . Let $K>0$. We have that

$$
\begin{aligned}
\mathbf{E}_{\theta} \xi=\mathbf{E}_{\theta} B(T)^{-1}(\kappa-S(T))^{+} & \leq \mathbf{E}_{\theta} B(T)^{-1} \kappa=e^{-K T} \kappa \mathbf{E}_{\theta} \widehat{B}(T)^{-1} \\
& =e^{-K T} \kappa \widehat{B}(0)^{-1} \rightarrow 0 \text { as } K \rightarrow+\infty .
\end{aligned}
$$

Let us prove statement (ii) of Theorem 3 . Let $K<0$. We have

$$
\begin{aligned}
\mathbf{E}_{\theta} \xi & =\mathbf{E}_{\theta} B(T)^{-1}(\kappa-S(T))^{+}=\mathbf{E}_{\theta}\left(B(T)^{-1} \kappa-\widetilde{S}(T)\right)^{+} \geq \mathbf{E}_{\theta} B(T)^{-1} \kappa-\mathbf{E}_{\theta} \widetilde{S}(T) \\
& =\kappa e^{-K T} \mathbf{E}_{\theta} \widehat{B}(T)^{-1}-\widetilde{S}(0)=\kappa e^{-K T} \widehat{B}(0)^{-1}-\widetilde{S}(0) \rightarrow+\infty \quad \text { as } \quad K \rightarrow-\infty
\end{aligned}
$$

This completes the proof of Theorem 3 .

Proof of Theorem 4. Let $\theta=\theta_{K}$ and $\widehat{B}(t)$ be such as defined in the proof of Theorem 3.

Let us prove statement (i) of Theorem 4 . We have that

$\mathbf{E}_{\theta} \xi=\mathbf{E}_{\theta} B(T)^{-1}(S(T)-\kappa)^{+}=\mathbf{E}_{\theta} \mathbb{I}_{\{S(T)>\kappa\}} B(T)^{-1}(S(T)-\kappa) \leq \mathbf{E}_{\theta} \mathbb{I}_{\{S(T)>\kappa\}} B(T)^{-1} S(T)$.

Let $\widehat{S}(t)=e^{K t} S(t)$. By the definitions, we have that

$$
\begin{aligned}
d S(t) & =S(t)\left(a d t+V^{\top} d W(t)+\widehat{V}^{\top} d W(t)\right) \\
& =S(t)\left(a d t-V^{\top} \theta d t-\widehat{V}^{\top} \theta d t+V^{\top} d W_{\theta}(t)+\widehat{V}^{\top} d W_{\theta}(t)\right) \\
& =S(t)\left((a-\widetilde{a}) d t-\left(K-\alpha+\rho+\widehat{\rho}^{2}\right) d t+V^{\top} d W_{\theta}(t)+\widehat{V}^{\top} d W_{\theta}(t)\right)
\end{aligned}
$$

and

$$
d \widehat{S}(t)=\widehat{S}(t)\left((a-\widetilde{a}) d t+\left(\alpha-\rho-\widehat{\rho}^{2}\right) d t+V^{\top} d W_{\theta}(t)+\widehat{V}^{\top} d W_{\theta}(t)\right) .
$$

It follows from the standard estimates for stochastic differential equations that

$$
\sup _{K} \mathbf{E}_{\theta_{K}}|\widehat{S}(T)|<+\infty
$$

for $\theta=\theta_{K}$; see, e.g., Chapter 2 in [24]. Hence $\mathbb{I}_{\{S(T)>\kappa\}}=\mathbb{I}_{\left\{\widehat{S}(T)>\kappa e^{K T}\right\}} \rightarrow 0$ a.s. as $K \rightarrow+\infty$. By the Lebesgue Dominated Convergence Theorem, it follows that $\mathbf{E}_{\theta} \xi \rightarrow 0$ as $K \rightarrow+\infty$. Hence statement (i) of Theorem 4 follows.

Let us prove statement (ii) of Theorem 4. For $K>0$, we have that

$$
\begin{aligned}
\mathbf{E}_{\theta} \xi & =\mathbf{E}_{\theta} B(T)^{-1}(S(T)-\kappa)^{+}=\mathbf{E}_{\theta}\left(\widetilde{S}(T)-B(T)^{-1} \kappa\right)^{+} \\
& \geq \mathbf{E}_{\theta} \widetilde{S}(T)-e^{-K T} \mathbf{E}_{\theta} \widehat{B}(T)^{-1} \kappa=\widetilde{S}(0)-e^{-K T} \widehat{B}(0)^{-1} \kappa \rightarrow S(0) \quad \text { as } \quad K \rightarrow+\infty .
\end{aligned}
$$


In addition, we have that

$$
\mathbf{E}_{\theta} \xi=\mathbf{E}_{\theta} B(T)^{-1}(S(T)-\kappa)^{+}=\mathbf{E}_{\theta}\left(\widetilde{S}(T)-B(T)^{-1} \kappa\right)^{+} \leq \mathbf{E}_{\theta} \widetilde{S}(T)=S(0) .
$$

This completes the proof of Theorem 4 .

Proof of Theorem 5. Let $K>0$, and $\vartheta=\vartheta_{K}=-K \eta_{\theta}+\theta$. By the Girsanov's Theorem,

$$
W_{\vartheta}(t)=W_{\theta}(t)-K \int_{0}^{t} \eta_{\theta}(s) d s
$$

is a Wiener process under $\mathbf{P}_{\vartheta}$. By the definitions,

$$
R_{\theta}=\int_{0}^{T} \eta_{\theta}(t)^{\top} d W_{\theta}(t)=K \int_{0}^{T}\left|\eta_{\theta}(t)\right|^{2} d t+\int_{0}^{T} \eta_{\theta}(t)^{\top} d W_{\vartheta}(t) .
$$

Let

$$
\begin{aligned}
& N_{1}(K) \triangleq \mathbf{E}_{\vartheta}\left(\int_{0}^{T}\left|\eta_{\theta}(t)\right|^{2} d t\right)^{2}, \\
& N_{2}(K) \triangleq \mathbf{E}_{\vartheta}\left(\int_{0}^{T} \eta_{\theta}(t)^{\top} d W_{\vartheta}(t)\right)^{2} .
\end{aligned}
$$

We have that

$$
\inf _{K>0} N_{1}(K)=\inf _{K>0} \mathbf{E}_{\vartheta}\left(\int_{0}^{T}\left(\left|\eta_{\theta}(t)\right|^{2} d t\right)^{2} \geq \underset{\omega}{\operatorname{essinf}}\left(\int_{0}^{T}\left|\eta_{\theta}(t, \omega)\right|^{2} d t\right)^{2}>0 .\right.
$$

By Proposition 2 and by assumptions on $U_{\theta}$, it follows that $\operatorname{ess}_{\sup } \operatorname{su}_{t, \omega}^{T}\left|\eta_{\theta}(t, \omega)\right|^{2} d t<+\infty$. Hence

$$
\sup _{K>0} N_{2}(K)=\sup _{K>0} \mathbf{E}_{\vartheta} \int_{0}^{T}\left|\eta_{\theta}(t)\right|^{2} d t \leq \underset{\omega}{\operatorname{ess} \sup _{\omega}} \int_{0}^{T}\left|\eta_{\theta}(t, \omega)\right|^{2} d t<+\infty .
$$

Hence $\mathbf{E}_{\vartheta} R_{\theta}^{2} \geq K^{2} N_{1}(K)-2 K \sqrt{N_{1}(K) N_{2}(K)}+N_{2}(K) \rightarrow+\infty$ as $K \rightarrow+\infty$. This completes the proof of Theorem 5 .

Proof of Theorem 6. Let $K>1$, and let $y(t)$ evolves as

$$
d y(t)=\eta_{\theta}(t)^{\top} d W_{\theta}(t), \quad y(0)=0 .
$$

Let $T_{K}=T \wedge \inf \left\{t>0: \int_{0}^{t} y(s)^{2} d s \geq K\right\}$. Let

$$
q(t)=q_{K}(t) \triangleq-K y(t) \frac{\eta_{\theta}(t)}{\left|\eta_{\theta}(t)\right|} \mathbb{I}_{\left\{t \leq T_{K}\right\}} .
$$

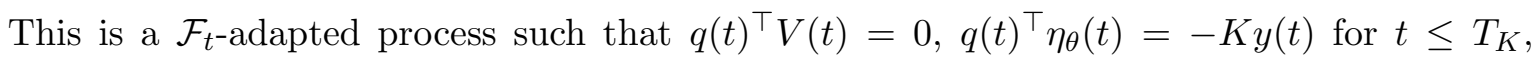
$q(t)=0$ for $t>T_{K},|q(t)| \leq K|y(t)|$, and

$$
\int_{0}^{T}|q(s)|^{2} d s=\int_{0}^{T_{K}}|q(s)|^{2} d s \leq K^{2} \int_{0}^{T_{K}} y(s)^{2} d s \leq K^{3} .
$$


In particular, it follows that $\theta-q \in \mathcal{T}$.

Let $\vartheta=\vartheta_{K} \triangleq \theta-q$. Then

$$
W_{\vartheta}(t)=W_{\theta-q}(t)=W_{\theta}(t)-\int_{0}^{t} q(s) d s
$$

is a Wiener process under $\mathbf{P}_{\vartheta}$. By the definitions, it follows that

$$
d y(t)=-K y(t) d t+\eta_{\theta}(t)^{\top} d W_{\vartheta}(t), \quad y(0)=0 .
$$

By Girsanov's Theorem, the measure $\mathbf{P}_{\vartheta}$ is equivalent to $\mathbf{P}_{\theta}$. We have that

$$
\begin{aligned}
R_{\theta} & =\int_{0}^{T} \eta_{\theta}(t)^{\top} d W_{\theta}(t)=\int_{0}^{T}\left[\eta_{\theta}(t)^{\top} q(t) d t+\eta_{\theta}(t)^{\top} d W_{\vartheta}(t)\right] \\
& =-\int_{0}^{T_{K}} K y(t) d t+\int_{0}^{T} \eta_{\theta}(t)^{\top} d W_{\vartheta}(t)=y\left(T_{K}\right)+\int_{T_{K}}^{T} \eta_{\theta}(t)^{\top} d W_{\vartheta}(t)
\end{aligned}
$$

By the assumptions on $U_{\theta}$ and by (13), it follows that

$$
\mathbf{C}_{\eta} \triangleq \underset{t, \omega}{\operatorname{ess} \sup }\left|\eta_{\theta}(t, \omega)\right|<+\infty
$$

Clearly,

$$
\begin{aligned}
& \mathbf{E}_{\vartheta} y\left(T_{K}\right)^{2}=\mathbf{E}_{\vartheta} \int_{0}^{T_{K}} e^{-2 K\left(T_{K}-s\right)}\left|\eta_{\theta}(s)\right|^{2} d s \leq \mathbf{C}_{\eta}^{2} \mathbf{E}_{\vartheta} \int_{0}^{T_{K}} e^{-2 K\left(T_{K}-s\right)} d s \\
& =\mathbf{C}_{\eta}^{2} \mathbf{E}_{\vartheta} \frac{1-e^{-2 K T_{K}}}{2 K} \leq \frac{1-e^{-2 K T}}{2 K} \rightarrow 0 \quad \text { as } \quad K \rightarrow+\infty .
\end{aligned}
$$

Consider events $A_{K}=\left\{\int_{0}^{T} y(t)^{2} d t>K\right\}=\left\{T_{K}<T\right\}$. We have

$$
\mathbf{E}_{\vartheta}\left(\int_{T_{K}}^{T} \eta_{\theta}(t)^{\top} d W_{\vartheta}(t)\right)^{2} \leq \mathbf{E}_{\vartheta} \int_{T_{K}}^{T}\left|\eta_{\theta}(t)\right|^{2} d t \leq E_{\vartheta} \mathbb{I}_{A_{K}} \int_{0}^{T}\left|\eta_{\theta}(t)\right|^{2} d t \leq T \mathbf{C}_{\eta}^{2} P_{\vartheta}\left(A_{K}\right) .
$$

We have that

$$
\mathbf{E}_{\vartheta} y(t)^{2} d t \leq \mathbf{C}_{\eta}^{2} \int_{0}^{t} e^{-2 K(t-s)} d s \leq \mathbf{C}_{\eta}^{2} T
$$

By the Markov Inequality and by (A.2), it follows that

$$
\mathbf{P}_{\vartheta}\left(A_{K}\right) \leq \frac{1}{K} \mathbf{E}_{\vartheta} \int_{0}^{T} y(t)^{2} d t \leq \frac{1}{K} \mathbf{C}_{\eta}^{2} T^{2} \rightarrow 0 \quad \text { as } \quad K \rightarrow+\infty .
$$

By (A.3)-(A.4), $\mathbf{E}_{\vartheta} R_{\theta}^{2} \rightarrow 0$ as $K \rightarrow+\infty$. This completes the proof of Theorem 6 .

Proof of Theorem \%. It can be verified directly that the equations for $S$ and $B$ have the desired form. 
Proof of Theorem 8. First, the standard Lagrange optimization techniques gives immediately that the selected $\theta$ is such that $|\theta(t, \omega)|$ is minimal over all $\theta \in \mathcal{T}$ and it is a unique solution of the problem

$$
\text { Minimize }|\theta| \text { subject to } V(t, \omega)^{\top} \theta=\widetilde{a}(t, \omega) \text {. }
$$

Further, by Martingale Representation Theorem, we have that, for some $U_{\theta} \in \mathcal{Y}_{\theta}$, presentation (10) holds. It was shown in Section 3 that (12)-(13) holds. By Proposition 1, we have that $V(t)^{\top} \eta_{\theta}(t) \equiv 0$. For our choice of $\theta$, this gives that $\theta(t)^{\top} \eta_{\theta}(t) \equiv 0$. It follows that

$$
R_{\theta}=\int_{0}^{T} \eta_{\theta}(t)^{\top} d W_{\theta}(t)=\int_{0}^{T} \eta_{\theta}(t)^{\top} d W(t)
$$

Hence

$$
\begin{aligned}
\mathbf{E} R_{\theta} \int_{0}^{T} \gamma(t) \widetilde{S}(t) V(t)^{\top} d W(t) & =\mathbf{E} \int_{0}^{T} \eta_{\theta}(t)^{\top} d W(t) \int_{0}^{T} \gamma(t) \widetilde{S}(t) V(t)^{\top} d W(t) \\
& =\mathbf{E} \int_{0}^{T} \gamma(t) \widetilde{S}(t) \eta_{\theta}(t)^{\top} V(t) d t=0 .
\end{aligned}
$$

This completes the proof of Theorem 8 .

Proof of Lemma 3 follows from the assumptions of Theorem 2 and from the equation

$$
B(T)^{-1}=B(t)^{-1} \exp \left(\left[-\alpha+\rho^{2} / 2+\theta_{1}\right](T-t)-\rho\left[W_{1 \theta}(T)-W_{1 \theta}(t)\right]\right) .
$$

Proof of Proposition 3. By the Itô formula, it follows that

$$
\begin{aligned}
& \xi-H(\mathrm{~s}(0), \mathrm{b}(0), 0)=H(\mathrm{~s}(T), \mathrm{b}(T), T)-H(\mathrm{~s}(0), \mathrm{b}(0), 0) \\
& =\int_{0}^{T}\left(H_{t}^{\prime}+H_{s}^{\prime}\left(-\widetilde{\sigma}^{2} / 2-\widetilde{\rho}^{2} / 2\right)+H_{b}^{\prime}\left(\alpha-\rho^{2} / 2-\widehat{\rho}^{2} / 2\right)+\mathcal{L} H\right) d t+\int_{0}^{T} U_{\theta}(t)^{\top} W_{\theta}(t),
\end{aligned}
$$

where

$$
U_{\theta}(t)=H_{s}^{\prime}(\mathrm{s}(t), \mathrm{b}(t), t) V(t)+H_{b}^{\prime}(\mathrm{s}(t), \mathrm{b}(t), t) \widehat{V}(t),
$$

and where $V(t)=V(\mathrm{~s}(t), \mathrm{b}(t), t), \widehat{V}(t)=\widehat{V}(\mathrm{~s}(t), \mathrm{b}(t), t)$. By the choice of $H$, it follows that

$$
\xi-H(\mathrm{~s}(0), \mathrm{b}(0), 0)=\int_{0}^{T} U_{\theta}(t)^{\top} W_{\theta}(t) .
$$

Hence $\mathbf{E}_{\theta} \xi=H(\mathrm{~s}(0), \mathrm{b}(0), 0)$ and (10) holds with this $U_{\theta}(t)$. We have established in Section 3 that

$$
\gamma(t)=\nu_{\theta}(t) \widetilde{S}(t)^{-1}, \quad \nu_{\theta}(t)=\frac{U_{\theta}(t)^{\top} V(t)}{|V(t)|^{2}}
$$


In addition, we have that

$$
\frac{U_{\theta}(t)^{\top} V(t)}{|V(t)|^{2}}=H_{s}^{\prime}(\mathrm{s}(t), \mathrm{b}(t), t)+H_{b}^{\prime}(\mathrm{s}(t), \mathrm{b}(t), t) \frac{\widehat{V}(t)^{\top} V(t)}{|V(t)|^{2}}
$$

This gives (38), since, by the assumptions of this section, we have that

$$
\frac{\widehat{V}(t)^{\top} V(t)}{|V(t)|^{2}}=\frac{(\widetilde{\sigma}(s, b, t) \rho(s, b, t)+\widetilde{\rho}(s, b, t) \widehat{\rho}(s, b, t))}{\widetilde{\sigma}(s, b, t)^{2}+\widetilde{\rho}(s, b, t)^{2}} .
$$

This completes the proof of Proposition 3.

Proof of Proposition 4. We have that

$$
\mathbf{E} R_{\theta}^{2}=\mathbf{E} \int_{0}^{T}\left|\eta_{\theta}(t)\right|^{2} d t, \quad \eta_{\theta}(t)=U_{\theta}(t)-\nu_{\theta}(t) V(t) .
$$

Under the assumptions of this section, $\eta_{\theta}(t)=g_{\theta}(\mathrm{s}(t), \mathrm{b}(t), t)$. Further, under the assumption of Proposition 1, $R_{\theta}=y(T)$, where the process $x(t)$ evolves as

$$
d y(t)=\eta_{\theta}(t)^{\top} d W_{\theta}(t)=\eta_{\theta}(t)^{\top} \theta(t) d t+\eta_{\theta}(t)^{\top} d W(t), \quad y(0)=0 .
$$

This can be rewritten as

$$
d y(t)=g_{\theta}(\mathrm{s}(t), \mathrm{b}(t), t)^{\top} \theta(\mathrm{s}(t), \mathrm{b}(t), t) d t+g_{\theta}(\mathrm{s}(t), \mathrm{b}(t), t)^{\top} d W(t) .
$$

This equation coupled with equations (35)-(36) describes evolution of a diffusion Markov process $(y(t), \mathrm{s}(t), \mathrm{b}(t))$ such that $J(y, s, b, t)$ is the solution in $\mathbf{R}^{3} \times[0, T]$ of the corresponding backward Kolmogorov-Fokker-Planck parabolic equation for the Markov diffusion process $(y(t), \mathrm{s}(t), \mathrm{b}(t))$,

By the Itô formula, it follows that

$$
\begin{aligned}
& \mathbf{E}\left[y(T)^{2}-J(y(0), \mathrm{s}(0), \mathrm{b}(0), 0)\right]=\mathbf{E}[J(y(T), \mathrm{s}(T), \mathrm{b}(T), T)-J(0, \mathrm{~s}(0), \mathrm{b}(0), 0)] \\
& =\mathbf{E} \int_{0}^{T}\left(J_{t}^{\prime}+J_{y}^{\prime} g_{\theta}^{\top} \theta+J_{s}^{\prime}\left(\widetilde{a}-\widetilde{\sigma}^{2} / 2-\widetilde{\rho}^{2} / 2\right)+J_{b}^{\prime}\left(r-\rho^{2} / 2-\widehat{\rho}^{2} / 2\right)+\mathcal{D} J\right) d t .
\end{aligned}
$$

By the choice of $J$, it follows that the right hand part of this equality is zero, i.e.

$$
\mathbf{E}\left[y(T)^{2}-J(0, \mathrm{~s}(0), \mathrm{b}(0), 0)\right]=0 .
$$

This completes the proof of Proposition 4.

\section{Acknowledgment}

This work was supported by ARC grant of Australia DP120100928 to the author. The author would like to thank the anonymous reviewers for the detailed suggestions that improved the manuscript. 


\section{References}

[1] K. Back, Martingale pricing, Annual Review of Financial Economics 2 (2010) 235-250.

[2] D. Becherer, The numeraire portfolio for unbounded semimartingales, Finance and Stochastics 5 (3) (2010) 327-341.

[3] S. Benninga, T. Bjork, and Z. Wiener, On the use of numeraires in option pricing. Journal of Derivatives 10 (2) (2002), 43-58.

[4] F. Biagini and M. Pratelli, Local risk minimization and numeraire. Journal of Applied Probability 36(4) (1999) 1126-1139.

[5] T.R. Bielecki, M. Jeanblanc, and M. Rutkowski. (2009). Credit Risk Modeling. Osaka University Press, Japan.

[6] M.J. Brennan, The role of learning in dynamic portfolio decisions, European Finance Review 1 (1998), 295-306.

[7] S.T. Cheng, On the feasibility of arbitrage-based option pricing when stochastic bond price processes are involved, Journal of Economic Theory 53(1) (1991) 185-198.

[8] N. Dokuchaev, Two unconditionally implied parameters and volatility smiles and skews. Applied Financial Economics Letters (2006) 2 199-204.

[9] N.G. Dokuchaev, Optimal solution of investment problems via linear parabolic equations generated by Kalman filter. SIAM J. of Control and Optimization 44 (4) (2005) $1239-1258$.

[10] N. Dokuchaev. (2012). Dynamic portfolio strategies: quantitative methods and empirical rules for incomplete information. Kluwer Academic Publishers, Boston.

[11] N. Dokuchaev, Option pricing via maximization over uncertainty and correction of volatility smile, International Journal of Theoretical and Applied Finance (IJTAF) 14 (4) (2011) 507-524.

[12] H. Föllmer and D. Sondermann, Hedging of non-redundant contingent claims. In: W. Hildenbrand and A. Mas-Colell (eds.), Contribution to Mathematical Economics (North Holland, New York, 1986) 205-223.

[13] H. Geman, N. El Karoui, and J.C. Rochet, Changes of numéraire, changes of probability measure and option pricing. Journal of Applied Probability 32(2) (1995), 443-458. 
[14] L. Y. Hin and N. Dokuchaev. (2014). On the implied volatility layers under the future risk-free rate uncertainty. International Journal of Financial Markets and Derivatives 3 (4), 392-408.

[15] L. Y. Hin and N. Dokuchaev. (2016a). Short rate forecasting based on the inference from the CIR model for multiple yield curve dynamics. Annals of Financial Economics, 11, No. 11650004 (33 pages).

[16] L. Y. Hin and N. Dokuchaev. (2016b). Computation of the implied discount rate and volatility for an overdefined system using stochastic optimization. IMA Journal of Management Mathematics, 27 (4), 505-527.

[17] A. Issaka and I. SenGupta. (2017). Analysis of variance based instruments for OrnsteinUhlenbeck type models: swap and price index, Annals of Finance 13 (4), pp 401-434 (2017).

[18] F. Jamshidian, Numeraire invariance and application to option pricing and hedging, working paper. http://mpra.ub.uni-muenchen.de/7167/, 2008

[19] M. Jeanblanc, M. Yor, M. Chesney. (2009). Mathematical Methods for Financial Markets, Springer. Heidelberg London New York.

[20] I. Karatzas and C. Kardaras. (2007). The numéraire portfolio in semimartingale financial models, Finance and Stochastics 11, 447-493.

[21] C. Kardaras (2010), Numéraire-invariant preferences in financial modeling, Ann. Appl. Probab. 20, 1697-1728.

[22] N. El Karoui and M. Quenez (1995), Dynamic programming and pricing of contingent claims in an incomplete market, SIAM Journal on Control and Optimization 33 (1) $29-66$.

[23] Yong-Jin Kim and Naoto Kunitomo, (1999). Pricing options under stochastic interest rates: A New Approach. Asia-Pacific Financial Markets 6 (1) 49-70.

[24] N.V Krylov. (1980), Controlled Diffusion Processes. Springer-Verlag. New York.

[25] J. Ruf (2013). Negative call prices. Annals of Finance 9 787-794.

[26] M. Schroder. (1999). Changes of numeraire for pricing futures, forwards, and options. Review of Financial Studies, 12(5):11431163. 
[27] M. Schweizer, A guided tour through quadratic hedging approaches, In: E. Jouini, J. Cvitanic, M. Musiela (eds.), "Option Pricing, Interest Rates and Risk Management", Cambridge University Press, 2001, 538-574.

[28] C. G. Turvey and S. Komar, Martingale Restrictions and the Implied Market Price of Risk, Canadian Journal of Agricultural Economics 54 (2006) 379-399.

[29] J. Vecer, and M. Xu (2004). Pricing Asian options in a semimartingale model, Quantitative Finance 4, 170-175.

[30] J. Vecer. (2011). Stochastic Finance: A Numeraire Approach, CRC Press. Boca Raton London New York.

[31] R. Weron, Market price of risk implied by Asian-style electricity options and futures, Energy Economics 30 (2008) 1098-1115.

[32] D. Won, G. Hahn, N. C. Yannelis. Capital market equilibrium without riskless assets: heterogeneous expectations. Annals of Finance (2008) 4, 183-195. 